\title{
Erratum
}

\section{The effect of exercise intensity and duration on the oxygen deficit and excess post-exercise oxygen consumption}

\section{J. Gore and R.T. Withers}

The Exercise Physiology Laboratory, School of Education, The Flinders University of South Australia, Bedford Park, South Australia 5042

In: $60: 169-174$

On page 170, left-hand column, in the section "Experimental design" point 3 should have read:

Consume a standardised pre-weighed meal before 2000 hours on the day preceding a test. Water only was drunk thereafter until a standardised pre-weighed light breakfast was consumed $3.5 \mathrm{~h}$ before any measurements were taken. 